International Journal of Engineering \& Technology, $7(2.31)(2018) 141-145$
International Journal of Engineering \& Technology
SPC
Website: www.sciencepubco.com/index.php/IJET
Research paper

\title{
DeyPoS-homomorphism authenticated tree (HAT) for multi-user locality in cloud storage
}

\author{
M. Muthu Selvam ${ }^{1}$, K. Mariappan ${ }^{2}$, G.V. Sriramakrishnan ${ }^{3}$, G. Suseendran ${ }^{4}$ \\ ${ }^{1}$ Assistant Professor, Department of IT, VISTAS, Chennai. \\ ${ }^{2}$ Assistant Professor, Department of IT, VISTAS, Chennai. \\ ${ }^{3}$ Assistant Professor, Department of IT, VISTAS, Chennai. \\ ${ }^{4}$ Assistant Professor, Department of IT, VISTAS, Chennai. \\ *Corresponding author E-mail:muthuselvam.mca@gmail.com
}

\begin{abstract}
The technology PoS (Dynamic Proof of storage) is a cryptographic primordial allows a abuser to test the reliability of subcontracted documents and effectively replace documents in the cloud storage system. Despite the fact that investigators have projected several dynamic proofs of storage designs in distinct client settings, hassle in the multi-client settings have now not been examined adequately. In sensible multi-client cloud server storage space wishes a cozy client part cross client system of deduplication, it permits client toward bypass importing manner as well as gain instantly the rights of the files, while different vendors of the same files hold uploaded to the cloud system server. In the direction of familiarity not a bit of prevailing dynamic Proof of Storages can guide this system. This research article we are bring the model of dynamic proof of storage in deduplicatable system and endorse a green creation known as Dedupicatable Dynamic Proof of Storage (DeyPoS), on the way to attain DeyPoS and comfy reduplication concurrently in cross client. Taking into account confront of formation assortment and personal blot creation make use of a new tool called HAT (Homomorphic Authenticated Tree). Also verify precautions of creation and the hypothetical, investigational outcomes shows that the creation is green in use.
\end{abstract}

Keywords: Homomorphic authenticated tree, DeyPoS, message authentication code, provable data possession, cloud storage service, data integrity

\section{Introduction}

In recent scenario storage subcontract is suitable and more striking to both academic side and industry area owing to the compensation of low price, lofty ease of access and effortless distribution. As one of the capacity outsourcing diagrams, distributed storage increments broad fixation in present day patterns [1] [2]. A few companies, for example, Microsoft, Yahoo and Google exhibit their own distributed storage administrations, wherever abusers can transfer their records to the servers, ideal to utilize them from an assortment of gadgets and disseminate them with others. Regardless of whether distributed storage administrations are widely acknowledged in introduce time in participation still stay heaps of security issues and conceivable dangers [3] [4]. A standout amongst the most critical properties is information honesty, when a customer contract out records to distributed storage. Customers ought to be affected that the records not put away in the server. Constant frameworks for protecting information respectability, similar to message validation codes (MACs) and furthermore advanced marks require clients to exchange every one of the documents from the cloud server for check that secures a huge correspondence Value [5]. The above said techniques are not adept for distributed storage benefits wherever abusers could try out the respectability as a rule [6]. In like manner, the analysts get Proof of Storage (PoS) [7] for looking at the trustworthiness while not downloading documents from the cloud server. Additionally, customers may likewise require numerous dynamic activities, similar to alteration, inclusion, and erasure, likewise to refresh their records, though maintaining the capability of PoS. Dynamic PoS [8] is made arrangements for such sort of powerful tasks. In qualification with PoS and dynamic PoS utilize each structure [9] like the Merkle tree [10]. Thusly, once the dynamic activities square measure dead, abusers recover labels which square measure utilized for respectability checking likes MACs and marks for the rebuilt pieces single, instead of make for every one of the pieces. To rose see the susequent substance; we tend to blessing a great deal of insights concerning PoS and dynamic PoS. In these plans [5] [8] [11] each square of a record is associated a cryptographic label that is utilized for strong the respectability of that piece. Once a supporter needs to discover the honesty of a document, it selfassertively chooses some square records of the document, and impels them to the cloud server.

As indicated by these go up against keys, the cloud server return the resulting hinders adjacent to their labels. The hero checks the piece respectability and record accuracy. The past is specifically secure by cryptographical labels. An approach to deal with the last is that the significant qualification amongst PoS and dynamic PoS. The a large portion of the plans of PoS [5] [11] [12] is the square list is prearranged its name, this infers the hero will try out the piece trustworthiness and furthermore record rightness. Then again, dynamic PoS can't compose the piece files into marks in light of the fact that the dynamic tasks may revision many records of non-supported obstructs that cause save working out and explanation esteem. Authentic structures are presented in powerful PoSs [8] [13] [14] to unwind this test. At long last the outcome appears, the labels are associated with each structure rather than the piece records. 


\section{Literature study}

Distributed computing is the vision of processing as an utility and has the inactive to improve an outsized a piece of the IT business, making programming framework even extra alluring as an administration and forming the approach IT equipment is implied and acquired. Engineers with imaginative thoughts for fresh out of the box new net administrations now not require the huge capital costs in equipment to send their administration or the human cost to work it. they have not struggle with respect to overprovisioning for an administration whose quality doesn't meet their expectations, in this manner squandering costly assets, or under-provisioning for one that turns out to be fiercely all around preferred, along these lines missing potential clients and income. Also, organizations with monster clump situated errands will get comes about as fast as their projects will scale, since misuse a thousand servers at one hour costs no very abuse one server for a thousand hours. This physical property of assets, while not paying a premium for huge scale is exceptional inside its historical backdrop [10].

Information honesty and capacity power are two essential requirements for distributed storage. POR (Proof of Irretrievability) and PDP (Proof of Data Possession) frameworks comfort information trustworthiness for distributed storage. The POW (Proof of possession) get betters stockpiling strength by solidly take away unnecessarily copied learning on the capacity server. In spite of the fact that immaterial gathering of two frameworks in order to understand every datum honesty and capacity strength prompts non-inconsequential duplication of information that is verification labels, to encourage can't help contradicting the motivation behind evidence of possession. Present day try to the present disadvantage present enormous methodology and correspondence costs and have furthermore been confirmed not secure. It requests a substitution determination to help sparing and secure information respectability reviewing with capacity deduplication for distributed storage. Amid this paper they tend to tackle this open disadvantage with a totally one of a kind topic upheld methods together with polynomial-based confirmation labels and homomorphic direct authenticators [5].

The distributed storage structures have turned out to be increasingly well known. A promising innovation that proceeds with their cost down is deduplication, which stores least difficult a solitary proliferation of rehashing measurements. Customer side deduplication endeavors to wind up mindful of deduplication conceivable outcomes as of now at the client and spare the data transfer capacity of bringing in duplicates of present records to the server. In this work we wind up mindful of strikes that exploit customer side deduplication, enabling an assailant to pick up get right of section to discretionary length records of various clients essentially in view of little hash marks of those reports. additional fundamentally, an assailant who knows about the hash mark of a record can convince the carport bearer that it possesses that report; consequently the server we could the aggressor download the entire document. To beat such assaults, we present the view of confirmations of possession (PoWs), which we could a client viably demonstrate to a server that that the client holds a report, rather than just a couple of snappy certainties around it. [6].

Information uprightness and capacity ability are the two critical requirements for distributed storage. Legitimate clients get to the information and circulate the documents in secured way. The CSS (distributed storage benefit) lessen the inconvenience for capacity administration and maintaining. Part Structure, arbitrary inspecting and record table is utilized to develop the Audit benefit. These systems are upheld provable updates to cloud outsourced information. The outsider examining permit to spare time and calculation assets with lessened online weight of the client. Probabilistic question and intermittent check for enhancing the execution of review administrations and furthermore review framework confirms the honesty [11]. The outsider examining permit to spare time and calculation assets with diminished online weight of the client [9]. In this work, a technique in view of
Probabilistic inquiry and occasional check for enhancing the execution of review administrations and furthermore review framework confirms the uprightness [3].

\section{Existing system}

Most of the current dynamic PoSs a tag is utilized for unwavering quality substantiation is making through the mystery key of the uploader. Along these lines, different proprietors who have the ownership of the report however have not transferred it as a result of the cross-client deduplication at the customer viewpoint can't make a shiny new tag once they refresh the report. For this situation the dynamic PoSs could come up short [1].

Halevi et al. included the idea of POW which is an answer of cross-client deduplication on the customer feature. It needs that the buyer can make the Merkle tree selective of the assistance from the cloud server that is an immense task in powerful PoS. Pietro and Sorniotti anticipated an alternate POW strategy advance the execution [2]. Xu et al. anticipated a supporter aspect deduplication strategy for encoded data; however the strategies make utilization of a deterministic confirmation calculation which shows that all document has deterministic brief proof. Therefore, everyone who acquires this verification can circumvent the affirmation without having the report locally [2].

The subsequent downsides are in the existing systems are

- The existing dynamic PoSs cannot be complete to the multi-user setting [1].

- Every existing technique for cross-client deduplication on the customer side is considered for static documents. On one event the records are refreshed the cloud server needs to reestablish the entire verified structures for these documents, which grounds profound calculation cost on the server-side [1].Owing to the difficulty of structure diversity and private tag generation, existing method cannot be complete to the dynamic PoS [1] [2].

- These methods unfortunately cannot sustain deduplication owing to structure diversity and private tag generation [1].

\section{Proposed structure}

To the best of our information, this can be the essential push to get a crude known as Dey Pos ( deduplicatable dynamic Proof of Storage)[2], that explains the structure assorted variety and individual label age challenges [2].

In refinement to the predominant bore witness to structures like skip rundown and Merkle tree. We tend to style a one of a kind bore witness to structure alluded to as HAT ( Homomorphic Authenticated Tree)[2] to downsize the correspondence esteem in each the confirmation of capacity segment and subsequently the deduplication area with comparable calculation esteem [2].Homomorphic Authenticated Tree will manage trustworthiness check, dynamic activities, and cross-client deduplication with sensible unwavering quality. We have a tendency to recommend and set in motion the essential conservative development of deduplicatable dynamic PoS alluded to as Dey-PoS, that backings boundless assortment of confirmation and refresh tasks. The assurance of this development is demonstrated inside the irregular prophet display, and consequently the execution is examined in principle and by experimentation [2].

The projected scheme furnish the following recompense

- It is an efficient authenticated structure.

- It is the primary realistic deduplicatable dynamic PoS method called DeyPoS and confirmed its safety in the random oracle model [2].

- The hypothetical and investigational consequences demonstrate that our deduplicatable dynamic PoS[2] execution is well-organized

- It plays better specifically whilst the report length and the wide variety of the challenged blocks are large [2] 


\section{Implementation}

In this investigation work we proposed the following modules

\section{User registration module}

During this module a user needs to transfer files in an exceedingly cloud server, First they ought to registration. Then solely they will be ready to be intimate. For that they have to fill the appropriate information within the registration area. All the information is sustained in a database file. In user registration module, the user have to be compelled to login, they must login by giving their user name and correct password.

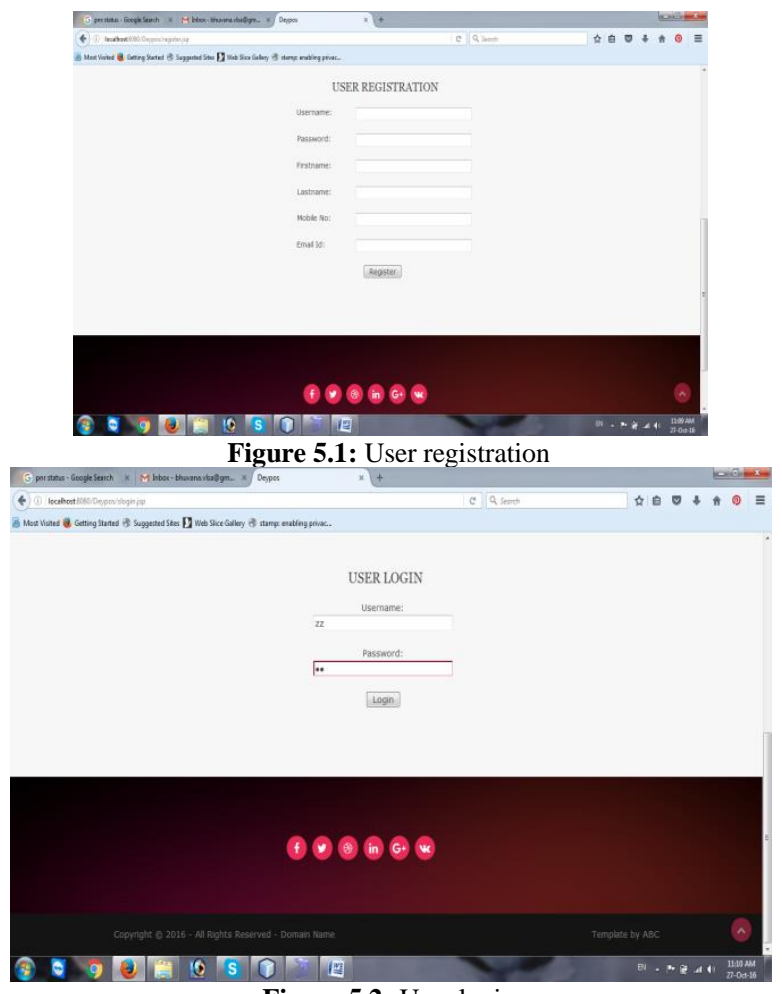

Figure 5.2: User login

\section{Procedures for upload/download files}

\section{Upload}

In this phase user upload their file then the uploaded file is changed into encrypted layout. In the encryption layout we are enforcing Bit changing method (BEM). The uploaded report isn't accumulated into the cloud server. The authorized assessor verifies the person record then only user documents is uploaded in to the cloud server [3].

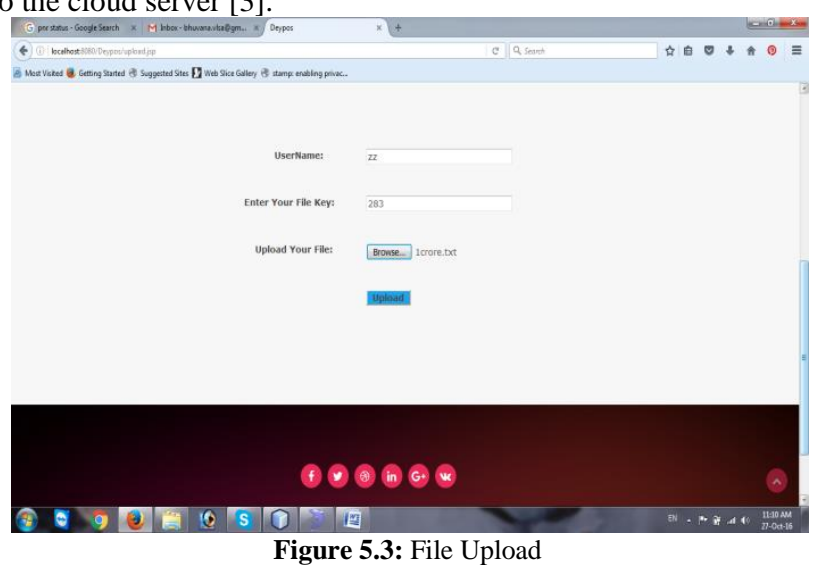

Download
In this phase decrypted format files download by the user before that the user must enter the appropriate encrypt key then only the file downloaded is decrypted. We are used BEM for decryption process.

\section{Structure for deduplication}

In block level deduplication the file is separated into blocks and ensures deduplication for the block. We are going to use Bit Exchanging Method for encryption. The byte and Block levels of information deduplication process bring the advantage of very good storage facility. While, during which and the way the techniques paintings ought to be reviewed to your facts backup surroundings and its specific needs prior choosing one technique over another. Deduplication of data can typically function at the file, block or byte stage for this reason significant least data fragment that is verified by way of redundancy for system. Hash method produces a distinctive identifier that is called hash number for all evaluated mass of data, then it is stock up in an index and notify duplicates that the duplicated wreckage have the identical hash numbers [3].

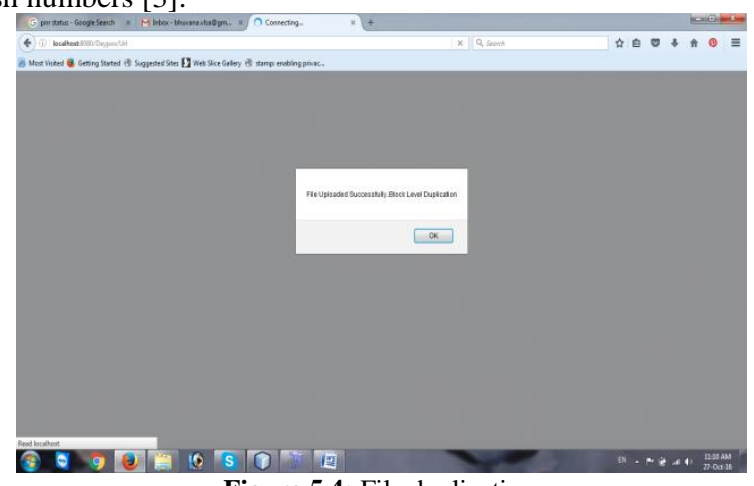

Figure 5.4: File duplication

\section{System of bit exchange}

By using simple bit shifting and XOR operation produces undisclosed key messages taken by encryption. Encrypting any file using bit exchange method [3].

Rules

- Fetch the byte one by one from the covert data and remodel every byte in to 8 bits. After that concern bit right shift operation [3].

- Next split the 8 bits into two modes and then carry out XOR operation with 4 bits on the left side and for right side 4 bits [3].

- Do the similar process recurring for all the bytes in file [3].

\section{Input design model and output DESIGN model}

\section{Input design model}

The input design model is that the bridge among the data system and therefore the user. It contains the plans and methods for knowledge grounding and these things are essential to place dealings knowledge in to a exploitable kind for process is attained by examining the $\mathrm{c}$ system to scan knowledge from a printed or in black and white document or it will take place by having user keying the information directly into the system. The look of input focuses on dominant the quantity of input needed, dominant the errors, delay avoidance, avoiding further steps and keeping the method straightforward. The input is meant in such the way so it provides security and simple use with retentive the privacy. Input style thought about the subsequent things

- Which kind of data is given as key input?

- What way the data is prearranged or implicit? 
- What are the techniques for set up input validations?

- What are the steps to follow for remove error?

The main Objectives of input designs are,

- The input design is that the method of changing a useroriented depiction of the input into a system based method. This style is very significant to evade errors within the information input method and illustrate the proper direction to the supervision for obtaining accurate data from the automated system

- This is accomplishing by making easy screens for the information entry to grip giant volume of information. The objective of planning input is to form knowledge entry easier and to be free from errors. The information entry screen is meant in such the simplest way that everyone the information manipulates is often performed. It conjointly present proof screening services.[4]

- While the information is entered it's going to take a look at for its legality. Records can be entered with the aid of displays. Suitable communications are given that as when wished so that the consumer will now not be in immediate. For that reason the goal of enter layout is to create an enter format that is simple to observe [4].
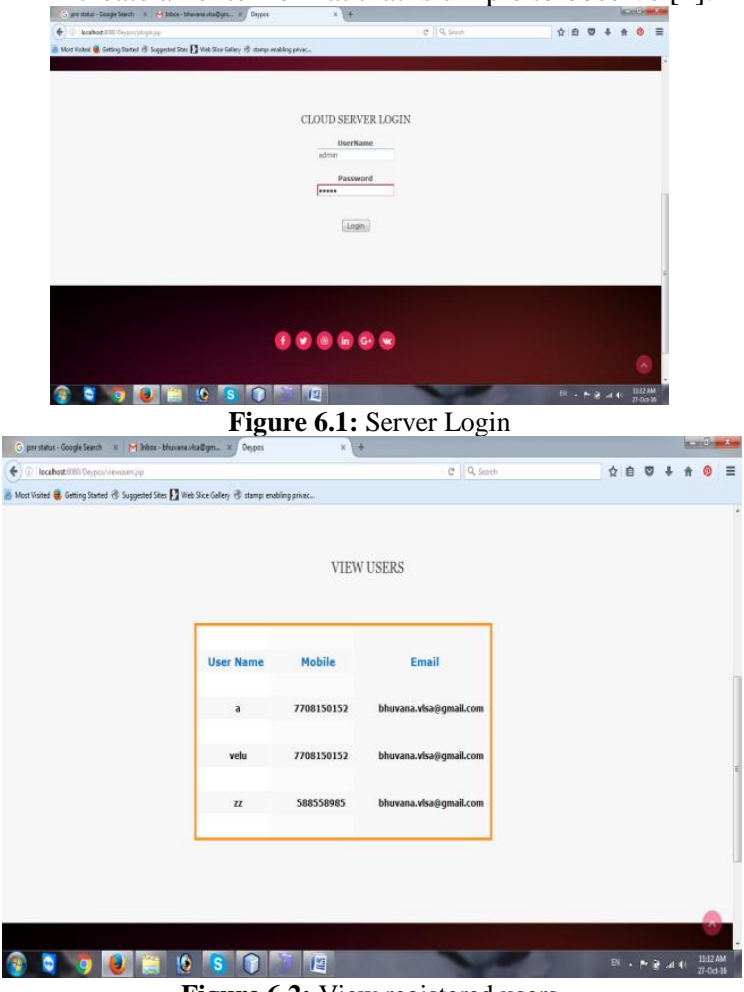

Figure 6.2: View registered users

\section{Output plan structure}

An eminenant output is one that meets the necessities of the top user and shows the data unmistakably. In a few system outcome of process are converse to the users and to alternative system in the course of outputs. In the output design structure it is resolute however the data is to be relocate for instantaneous would like and conjointly the text output. It is the foremost necessary and straight supply information to the user. Economical and clever output design structure get better the system's connection to assist user take decision [8].

- Manipulating system output have to progress in an prearranged, nicely concept out way; the exact output have to be advanced while making sure that all output component is considered in order that human beings will locate the device can use without difficulty and efficaciously. While investigation proposes system output, they have to pick out the precise output that is needed to meet the necessities.

- Choose techniques for demonstrate details.

- Generate manuscript, description, or other set-up that hold data created by the

Computer system [8].

The productivity shape of an information method should achieve more than one of the subsequent objectives. [8]

- deliver information statistics approximately beyond activities, cutting-edge reputation or projections of the future.[8]

- Indicate crucial occasions, prospects, issues, or cautions.[8]

- $\quad$ Eelicit an movement.[8]

- $\quad$ verify an movement.[8]

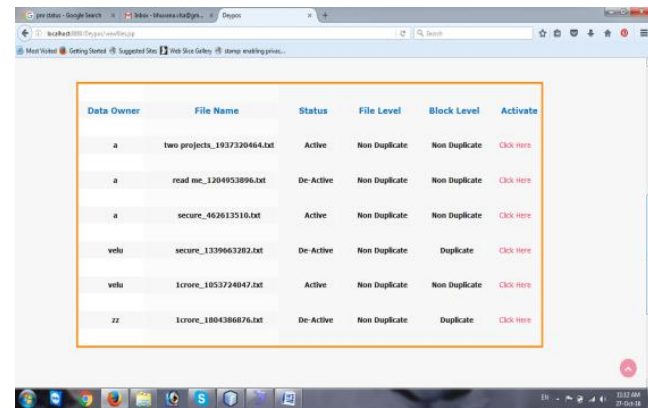

Figure 6.3: View Files

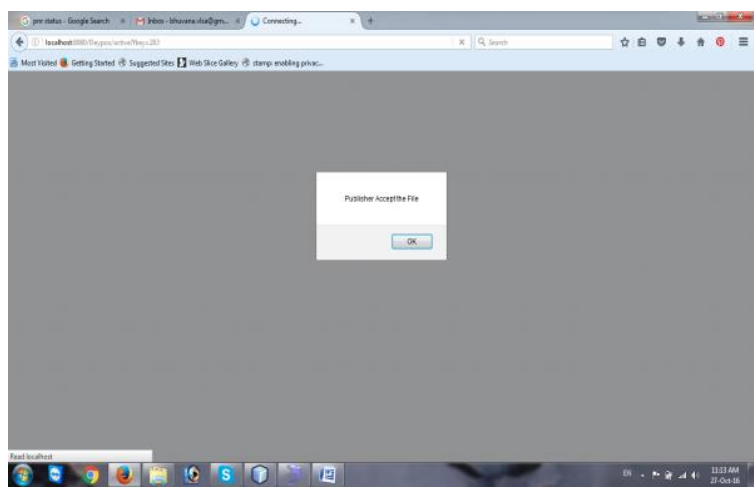

Figure 6.4: Server Accept File

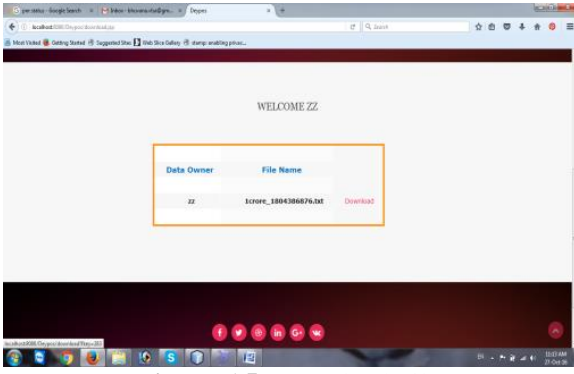

Figure 6.5: Download File

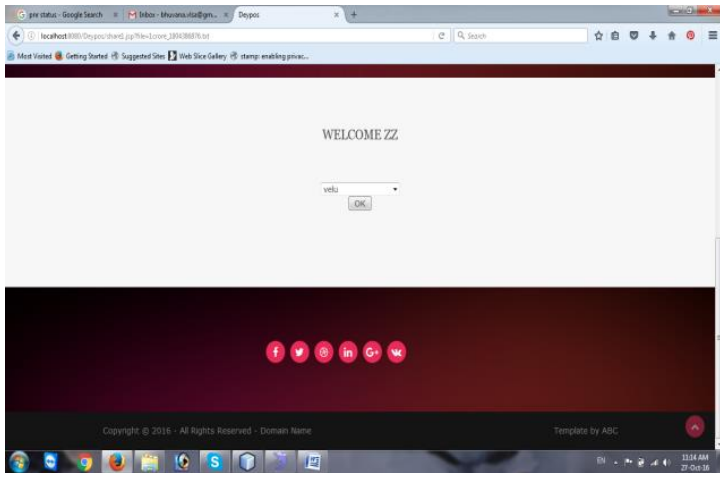

Figure 6.6: Distribute Files 

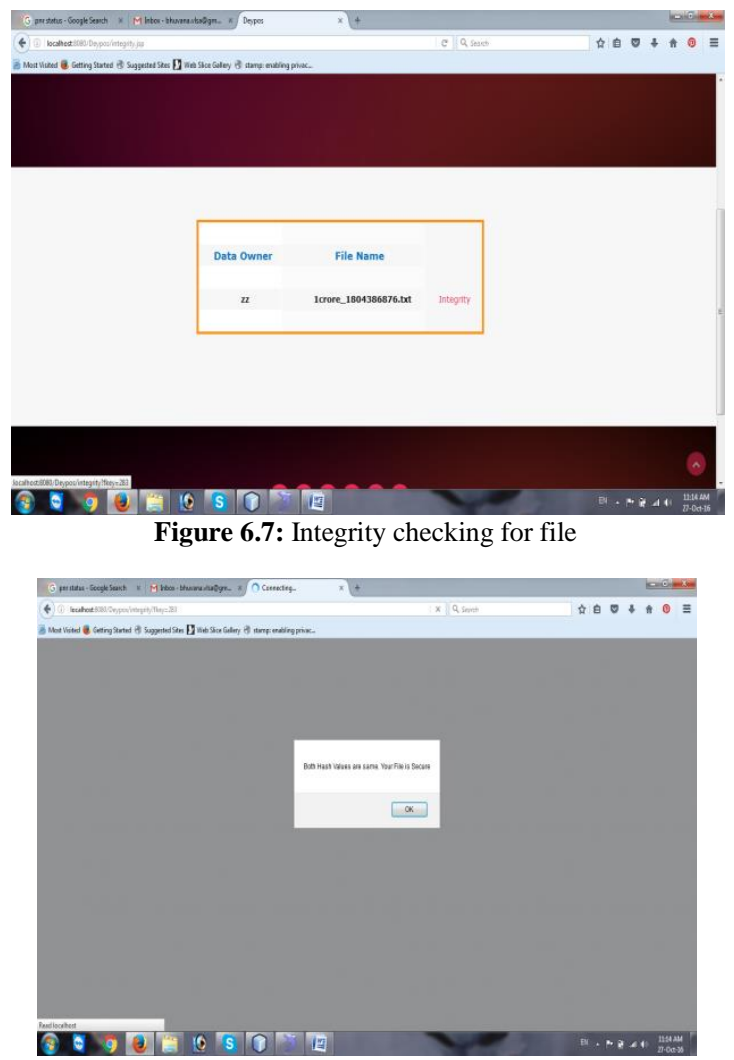

Figure 6.8: Hash value matching

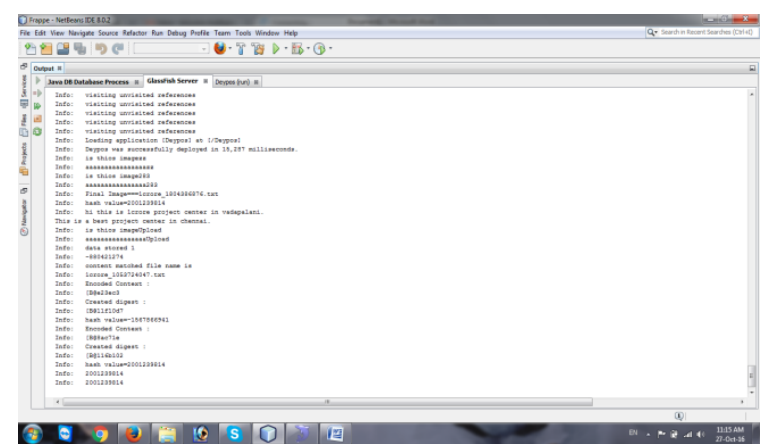

Figure 6.9: Output

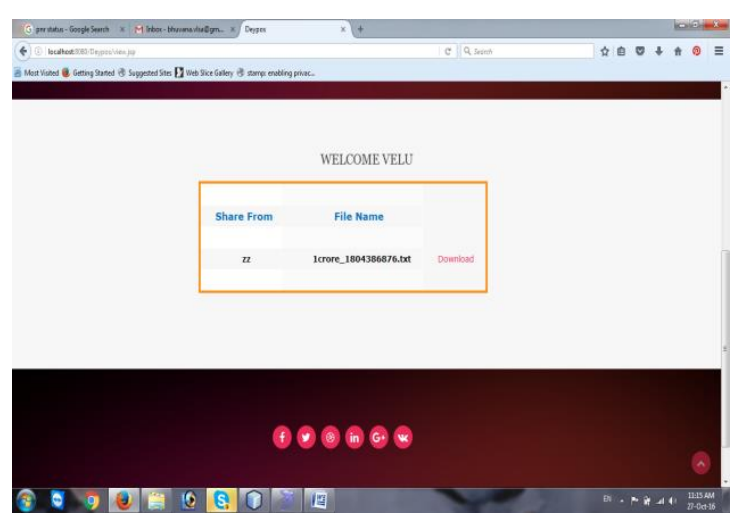

Figure 6.10: View received files

\section{Conclusion}

We projected the complete prerequisites in multi-person cloud storage structures and delivered the version of deduplicatable dynamic PoS (DeyPos) [7]. We planned a singular device known as HAT which is an effective legitimate formation [7].

We anticipated the primary matter-of-fact deduplicatable dynamic PoS system known as DeyPoS and attested its safety inside the random oracle version. The hypothetical and investigational outcomes display to facilitate our DeyPoS execution is competent, more than ever whilst the record length and the wide variety of the confront blocks are outsized [7].

\section{References}

[1] Kamara S \& Lauter K, "Cryptographic cloud storage", Proc. of FC, (2010)

[2] Xia Z, Wang X, Sun X \& Wang Q, "A Secure and Dynamic MultiKeyword Ranked Search Scheme over Encrypted Cloud Data", IEEE Transactions on Parallel and Distributed Systems, Vol.27, No.2, (2016), pp. 340-352.

[3] Xiao Z \& Xiao Y, "Security and privacy in cloud computing", IEEE Communications Surveys Tutorials, Vol.15, No.2, (2013), pp.843-859.

[4] Ardagna CA, Asal R, Damiani E \& Vu QH, "From Security to Assurance in the Cloud: A Survey", ACM Comput. Surv., Vol.48, No.1, (2015), pp.2:1-2:50.

[5] Ateniese G, Burns R, Curtmola R, Herring J, Kissner L, Peterson Z \& Song D, "Provable data possession at untrusted stores", Proc. of CCS, (2007), pp.598-609.

[6] Ateniese G, Di Pietro R, Mancini LV \& Tsudik G, "Scalable and Efficient Provable Data Possession", Proc. of SecureComm, (2008)

[7] Ateniese G, Kamara S \& Katz J, "Proofs of storage from homomorphic identification protocols", Proc. of ASIACRYPT, (2009), pp. 319-333.

[8] Erway C, K"upc "u A, Papamanthou C \& Tamassia R, "Dynamic provable data possession", Proc. of CCS, (2009), pp.213-222.

[9] Tamassia R, "Authenticated Data Structures", Proc. of ESA, (2003), pp.2-5.

[10] Wang Q, Wang C, Li J, Ren K \& Lou W, "Enabling public verifiability and data dynamics for storage security in cloud computing", Proc. of ESORICS, (2009), pp.355-370.

[11] Armknecht F, Bohli JM, Karame GO, Liu Z \& Reuter CA, "Outsourced proofs of retrievability", Proc. of CCS, (2014), pp. 831-843.

[12] Shacham H \& Waters B, "Compact Proofs of Retrievability", Journal of Cryptology, Vol.26, No.3, (2013), pp.442-483.

[13] Mo Z, Zhou Y \& Chen S, "A dynamic proof of retrievability (PoR) scheme with o(logn) complexity", Proc. of ICC, (2012), pp.912916.

[14] Shi E, Stefanov E \& Papamanthou C, "Practical dynamic proofs of retrievability", Proc. of CCS, (2013), pp.325-336.

[15] Halevi S, Harnik D, Pinkas B \& Shulman-Peleg A, "Proofs of ownership in remote storage systems", Proc. of CCS, (2011), pp. 491- 500.

[16] Douceur J, Adya A, Bolosky W, Simon P \& Theimer M "Reclaiming space from duplicate files in a serverless distributed file system", Proc. of ICDCS, (2002), pp. 617-624.

[17] Juels A \& Kaliski, Jr BS, "PORs: Proofs of retrievability for large files", Proc. of CCS, (2007), pp.584-597.

[18] Shacham H \& Waters B, "Compact proofs of retrievability", Proc. of ASIACRYPT, (2008), pp.90-107. 